\title{
DILUTED MAGNETIC SEMIMETALS - BEYOND HEISENBERG COUPLING
}

\author{
Z. WILAMOWSKI, T. STORY \\ Institute of Physics, Polish Academy of Sciences \\ Al. Lotników 32/46, 02-668 Warszawa, Poland \\ AND W. JANTSCH \\ Johannes Kepler University, 4040 Linz, Austria
}

We analyse effects which cannot be explained assuming the isotropic Heisenberg form of $s p-d$ or of the indirect $d-s p-d$ (RKKY) coupling. Resonance line broadening, a line shift and an enhancement of the magnetic anisotropy in diluted magnetic semimetals are discussed.

PACS numbers: 75.20. $-\mathrm{g}, 75.30 . \mathrm{Hx}, 76.20 .+\mathrm{q}$

The $s p-d$ coupling between localized $(d)$ spins, $S$, and the spin $\sigma$ of conduction $(s p)$ electrons usually is described by the symmetric Kondo Hamiltonian, $a_{k^{\prime}}^{+} a_{k}^{-} \sigma S$, or, equivalently, by the Heisenberg form of the spin Hamiltonian, $J_{s p-d} \sigma S$. This approximation is sufficient for a description of most basic effects, which are caused by $s p-d$ coupling. Such effects are, e.g., spin-flip scattering and the Knight-like $g$-shift of the impurity resonance. Spin flip scattering causes a spin polarization of the conduction band electrons (CE) which, within second order perturbation, leads to the indirect $d-s p-d$ (RKKY) exchange coupling among the impurity spins.

The character of the spin polarization is different in semiconductors and in semimetals. In semimetals, the spin polarization is localized in the vicinity of the localized $d$-state and decreases with a decay length $\left(2 k_{F}\right)^{-1}$, as described by the Ruderman-Kittel (RK) function. The spatial integral of spin polarization yields the magnetic moment induced in the conduction electron gas by the local spin: $\sigma_{\text {ind }}=(3 / 16)\left(J_{s p-d} n_{0} / E_{\mathrm{F}}\right)\left(n_{\mathrm{CE}} / n_{0}\right) S$, where $E_{\mathrm{F}}$ is the Fermi energy, $n_{0}$ is concentration of crystal sites and $n_{\mathrm{CE}}-$ that of the CE. The sum of the induced magnetic moments determines the magnetization of electron gas caused by the presence of the local spins. The resulting magnetization value is, however, only half of the value obtained within the one-electron approach, commonly used for semiconductors. Within the latter approach, the spin polarization of the CE is assumed to be homogeneously distributed in space and it causes an effective $g$-factor enhancement [1]. 
The effects discussed above are caused by the isotropic, i.e. IIeisenberg-like, $s p-d$ and $d-s p-d$ couplings. Non-Heisenberg terms are usually much weaker and they do not influence these effects strongly. There is, however, a class of phenomena which originate from non-Heisenberg spin interactions only. In particular, it is known that the Heisenberg coupling between similar spins leads neither to a shift of the magnetic resonance nor to any modification of the second moment of the resonance line. Magnetic resonance spectroscopy on diluted magnetic semiconductors thus is strongly affected by non-Heisenberg spin interactions and therefore it constitutes an effective tool for investigations of spin coupling details. Especially interesting results are obtained for diluted magnetic (DM) semimetals, whose spin coupling reflects the complexity of the band structure, of the Fermi surface, and of the microscopic details of the spatial dependence of the $s p-d$ coupling.

In this paper, we present experimental evidence for the role of the conduction electron gas in semimetals. We consider: (i) non-Heisenberg RKKY coupling, i.e. non-isotropic components of the second order $d-d$ coupling, known as the pseudo dipole-dipole, and the Dzialoshynski-Moriya coupling; (ii) higher order indirect $d-d$ coupling, where the polarization of the electron gas, due to saturation must be described by a higher than second order susceptibility tensor; and (iii) effects which arise from the interference of the $s-p d$ exchange coupling and magnetic anisotropy.

The importance of non-Heisenberg $d-s p-d$ coupling is clearly evident from the dependence of the EPR linewidth on the electron and the impurity concentration. A systematic analysis of this effect in Mn-based DM semimetals is difficult because of the rich structure of Mn spectra and a strong overlap of the fine and the hyperfine structures. Moreover, the concentration of the conduction electrons in the Mn compounds cannot be easily varied. More valuable experimental data can be obtained in mixed valence systems, where the electron concentration can be effectively changed by doping: in $\mathrm{HgSe}: \mathrm{Fe}$ up to $5 \times 10^{18} \mathrm{~cm}^{-3}$, in $\mathrm{PbTe}$ :Cr up to $1.3 \times 10^{19} \mathrm{~cm}^{-3}$ and in PbSe:Cr up to $1.7 \times 10^{19} \mathrm{~cm}^{-3}$,

The temperature dependence of the EPR linewidth in $\mathrm{HgSe}: \mathrm{Fe}$ is shown in the inset of Fig. 1. At high temperatures, Korringa broadening manifests itself by its linear temperature dependence. At lower temperature, the linewidth saturates - obviously another mechanism dominates there. The fact that it is temperature independent indicates that this broadening is caused by a transverse relaxation time. Such a behavior is typical of dipole-dipole coupling. The dipolar coupling is, however, at least by two orders of magnitude too weak to explain the line broadening observed here. Therefore we suggest that the observed spin relaxation is governed by the non-Heisenberg components of the RKKY coupling.

In fact, the efficiency of the broadening mechanism depends on the carrier concentration. The experimentally observed parabolic dependence of the linewidth on the electron concentration, $\Delta H \propto n_{\mathrm{CE}}^{2}$ (see Fig. 1) supports this supposition. The RKKY coupling scales with the square of the density of conduction band states at the Fermi level, $\rho\left(E_{\mathrm{F}}\right)^{2} \propto n_{\mathrm{CE}}^{2 / 3}$. In addition, the linewidth is expected to be proportional to the concentration of spins in $\mathrm{HgSe}: \mathrm{Fe}$ which equals the electron concentration, $n_{\mathrm{CE}}$. Summarizing, within a parabolic approximation of conduction 


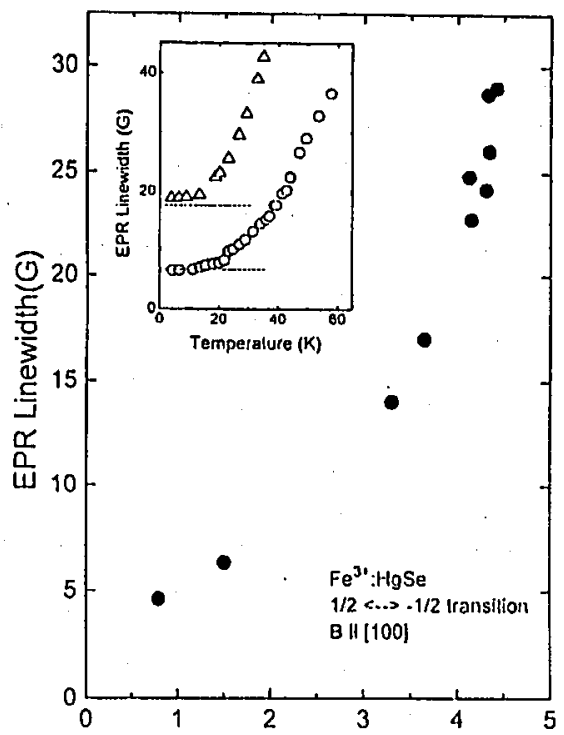

Fig.1 Electron Concentration $\left(10^{18} \mathrm{~cm}^{-3}\right)$

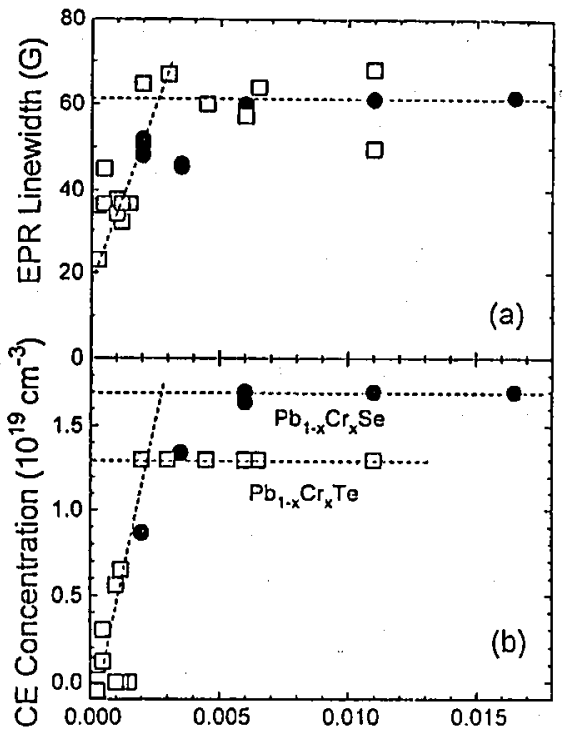

Fig. 2 Cr Composition, $x$

Fig. 1. Dependence of the low temperature linewidth of $\mathrm{Fe}^{3+}$ in $\mathrm{HgSe}$ on the electron concentration. The inset shows the temperature dependence of the linewidth where the contributions of the temperature dependent Korringa broadening and temperature independent pseudo-dipole broadening are seen.

Fig. 2. Dependence of the low temperature linewidth (a) and electron concentration (b) on the $\mathrm{Cr}$ content in $\mathrm{PbTe}$ and $\mathrm{PbSe}$.

band, the linewidth caused by the non-Heisenberg component of RKKY coupling should scale with $n_{\mathrm{CE}}^{5 / 3}$. The experimentally observed exponent $2.0 \pm 0.2$ is slightly bigger, and the difference can be attributed to band non-parabolicity.

In Fig. 2a, the dependence of the EPR linewidth on the impurity content, $x$, is shown for lead chalcogenides doped with Cr. For low doping level, the increase in the impurity concentration (see Fig. 2b) is accompanied by an increase in both the electron concentration and the EPR linewidth. Upon further doping, the Fermi energy reaches the impurity level and both the electron concentration and the concentration of the $\mathrm{Cr}^{3+}$ ions saturate [2]. The increase in the concentration of the $\mathrm{Cr}^{2+}$ ions which takes place in this composition range does not lead to any modification of the linewidth. The spread of the experimental points originates from additional uncontrollable electrically active defects. A reexamination of other experimental data shows that the discussed mechanism of spin relaxation is quite common, albeit not so easy to investigate. In all semimetallic compounds, the resonance is strongly broadened. In real metals, this mechanism is expected to be still much stronger and to lead to a strong damping of the resonance.

Another class of effects which cannot be analysed within the Heisenberg scheme is that of magnetic anisotropy which manifests itself in the angular dependence of some ferromagnetic resonance (FR) [3] or that of the fine structure 
of some EPR spectrum [4]. The relation of the characteristic times in semimetals implies, however, a basic difference in ferro- and paramagnetic resonances. In both magnetic phases, the mean value of the induced magnetic moment, $\sigma_{\text {ind }}$, is similar. In the case of a paramagnetic resonance, however, the rate of the scattering events, which induces the mean moment of $\mathrm{CE}$, is much lower than the resonance frequency. Moreover, after the spin-flip scattering process the precession frequency of the local spin changes, contributing then to another fine structure line. As a consequence, EPR line broadening is the only effect of spin-flip scattering. Neither the frequency nor the fine structure is affected by the local spin polarization (the Knight shift is a consequence of the Pauli polarization of the CE, but not a consequence of the local polarization of the RK type). Well resolved fine structure has indeed been observed in semimetallic $\mathrm{HgSe}: \mathrm{Fe}$ [4].

The experimental findings are completely different in RKKY ferromagnets. The resonance field is affected by the concentration of $\mathrm{CE}$ and by the magnetization of the whole system. Also a strong enhancement of the "crystal field" anisotropy is observed [5]. These effects can be explained by considering the FR as a precession of two classically coupled moments: the total moment of the local spins and the magnetization of the $\mathrm{CE}$, which is equal to the sum of the induced moments, $\sigma_{\text {ind }}$. Coupling of the two macroscopic magnetic and mechanical moments with different $g$-factors results in a modification of the resonance frequency of the local spins. For ferromagnetic PbMnSnTe the estimation shows that about $1 \%$ of $\mathrm{CE}$ spins are uncompensated but because of the large $g$-factor of the conducting electrons this polarization is sufficient to explain the observed resonance shift.

An even more pronounced consequence of the moment coupling is the enhancement of the magnetic anisotropy. The anisotropic energies of coupled moments can be treated additively. Since the magnetic anisotropy of the CE is big, even a small contribution of the CE moment can cause a strong change of the anisotropy, as compared to the pure crystal field anisotropy of the local spins. Discussing anisotropy of CE one has to consider the diamagnetic anisotropy caused by the non-spherical shape of the Fermi surface. In the case of a DM semimetal, also the anisotropy of the paramagnetic susceptibility enters. In cubic crystals, the paramagnetic Pauli susceptibility is isotropic only, but the strong local spin polarization of $\mathrm{CE}$, much greater than the mean value, requires consideration of a higher order CE susceptibility, which is no more spherically symmetric. Higher order effects, caused by saturation of the $\mathrm{CE}$ polarization can also shift the resonance frequency.

\section{References}

[1] J. Furdyna, J. Appl. Phys. 64, R29 (1988).

[2] T. Story, Z. Wilamowski, E. Grodzicka, B. Witkowska, W. Dobrowolski, Acta Phys. Pol. A 84, 773 (1993).

[3] T. Story, P.J.T. Eggenkamp, C.H.W. Swüste, H.J.M. Swagten, W.J.M. de Jonge, A. Szczerbakow, Phys. Rev. B 47, 227 (1993).

[4] Z. Wilamowski, A. Mycielski, W. Jantsch, G. Hendorfer, Phys. Rev. B 38, 3621 (1988).

[5] P.J.T. Eggenkamp, T. Story, C.H.W. Swüste, H.J.M. Swagten, W.J.M. de Jonge, Acta Phys. Pol. A 84, 641 (1993). 